LAWRENCE LIVERMORE N A T IO N A L LABORATORY
Implications of Convective Scrape-Off Layer Transport for Fusion Reactors with Solid and Liquid Walls

M. Kotschenreuther, T. D. Rognlien, P. Valanju

November 14, 2003

Fusion Engineering and Design 
This document was prepared as an account of work sponsored by an agency of the United States Government. Neither the United States Government nor the University of California nor any of their employees, makes any warranty, express or implied, or assumes any legal liability or responsibility for the accuracy, completeness, or usefulness of any information, apparatus, product, or process disclosed, or represents that its use would not infringe privately owned rights. Reference herein to any specific commercial product, process, or service by trade name, trademark, manufacturer, or otherwise, does not necessarily constitute or imply its endorsement, recommendation, or favoring by the United States Government or the University of California. The views and opinions of authors expressed herein do not necessarily state or reflect those of the United States Government or the University of California, and shall not be used for advertising or product endorsement purposes. 


\title{
Implications of Convective Scrape-Off Layer Transport for Fusion Reactors with Solid and Liquid Walls
}

\author{
M. Kotschenreuther ${ }^{\mathrm{a}^{*}}, \mathrm{~T}$. Rognlien $^{\mathrm{b}}, \mathrm{P}$. Valanju $^{\mathrm{a}}$
}

a) Institute for Fusion Studies, University of Texas at Austin, TX 78712

b) Lawrence Livermore National Laboratory, Livermore, California 94551

\begin{abstract}
Recent experimental observations in tokamaks indicate enhanced convection of plasma blobs toward the main chamber wall. Potential implications of these observations for reactors are examined here. Two dimensional plasma edge calculations are performed with UEDGE, including convective transport consistent with present experiments. This is coupled to a kinetic neutral calculation using the code NUT, to compute the hot neutral flux to the wall. The inclusion of convection increases sputtering of the wall by roughly an order of magnitude. For tungsten walls, erosion (neglecting re-deposition) is estimated to be $\sim 0.6 \mathrm{~mm}$ per year. Plasma contamination could be serious for high $\mathrm{Z}$ walls of $\mathrm{W}$ or $\mathrm{Sn}$, and might preclude ignition (based on empirical screening estimates). Low $\mathrm{Z}$ liquid materials offer much better prospects for acceptable plasma contamination. Rough estimates of dust generation from such erosion rates imply significant safety issues. Plasma transport via blobs can also significantly modify models of impurity redeposition.
\end{abstract}

\section{Introduction}

It is widely appreciated that plasma facing components are a serious design challenge for thermonuclear reactors. These challenges will be compounded by any plasma behavior which increases the plasma interaction with the walls. Recent experimental results in some tokamaks reveal an unexpected phenomenon- plasma transport in the Scrape Off Layer (SOL) increases strongly with distance from the separatrix [1-3]. The large transport in the far SOL increases the plasma flux to the wall. To quote reference [1], "This observation has potentially important consequences for a reactor: contrary to the ideal picture of divertor operation, a tightly baffled divertor may not offer control of the neutral density in the main chamber such that charge exchange heat losses and sputtering of the main chamber walls can be reduced." Here, we present the first calculations of the potential impacts of large transport in the far SOL on reactors.

Main chamber erosion for reactor level devices has been computed before, using state of the art 2-D edge simulation codes to calculate the edge plasma profiles, and kinetic neutral simulation codes to calculate the neutral flux on the wall [4,5]. However, these 
investigations did not include a strong increase in plasma transport in the far SOL, as has been inferred recently. Rather, they used a conventional model where the SOL plasma diffusion is taken to be a constant in space. The code UEDGE [6] has been used to examine the far SOL of C-mod [1] and DIII-D [7]. With a conventional model, where the SOL diffusion is constant in space, quantities which indicate the degree of plasma interaction with the main chamber wall are underpredicted by up to an order of magnitude [7]. Such quantities include the far SOL plasma density profile, the $D_{\square}$ recycling light, and the neutral pressures at the outboard wall. Experimental results for these quantities are reproduced by UEDGE only when transport strongly increases with distance from the separatrix.

We include such transport here to estimate the consequences for a reactor. In principle, present experiments could be explained by invoking an increase in either convection or diffusion in the edge. However, there are experimental observations of rapid 'blob-like' convection [2,8-9] and physics models [10-12] which qualitatively reproduce the required speed and other characteristics of the blob motion. Conversely, there are no physics models which predict the extremely large diffusion required. A convection model is adopted in this work. However, we believe that an experimentally benchmarked diffusive model of the far SOL would give qualitatively similar results to a convection model.

The inability to confidently project the plasma transport processes in the SOL to a reactor is a longstanding problem in divertor design. Of necessity, an empirical approach is used in investigations of SOL transport in reactor scale devices. Diffusion coefficients are chosen based on values which are found to match present experimental results. We use a similar procedure to examine the effects of SOL convection in reactors, choosing convection values similar to those which fit present experimental results. Including such convection, the 2-D code UEDGE is used to obtain the plasma profiles. Characteristics of the convection model are varied to produce density profiles for reactor simulations with a relatively flat decay in the far SOL, as found in experiments.

As a further consistency check, the plasma flux to the wall is compared to experimental values and projections. We conclude that the inclusion of convection in UEDGE gives a more plausible reactor projection for main chamber plasma interaction than simulations using the conventional SOL model with only a constant diffusion coefficient.

Neutral dynamics are evaluated in UEDGE using a fluid model, which suffices to describe the bulk plasma-neutral interaction. However, it is well known that the neutralwall interaction, i.e. sputtering, can be dominated by high energy neutrals which cannot be described in such a fluid treatment. Using the plasma profiles and wall recycling source from UEDGE, the energy spectrum of the charge exchange neutral flux on the wall is computed using the kinetic neutral transport code NUT [13]. This code solves the full non-local, kinetic integral equations using a rapid solution algorithm and has been benchmarked with experiments $[14,15]$. Sputtering is predominantly from the high energy $\mathrm{C}$-X neutrals for high $\mathrm{Z}$ wall materials (e.g. W), whereas for low $\mathrm{Z}$ materials, both sputtering and direct plasma interaction are important. 
When convection is included, sputtering rates are increased by $\sim 4-10$ above cases without convection, such as past estimates. This is not surprising, since simulations without convection underestimate $\mathrm{D}_{\square}$ (i.e., the neutral source) by an order of magnitude. In the case of high $\mathrm{Z}$ tungsten, most of the sputtering arises from high energy neutrals generated inside the plasma. The increase in the edge neutral source also increases the plasma edge density, somewhat shielding the hot interior of the plasma from neutrals generated at the edge. Thus, the high $\mathrm{Z}$ sputtering is increased by a smaller factor than the increase in the neutral source.

Nonetheless, the potential implications of the increased sputtering are substantial. Impurity contamination of the plasma could be serious for the high $\mathrm{Z}$ materials. For impurity penetration factors in the empirical range, the increased tungsten source could preclude reactor operation due to excessive plasma cooling by radiation. If $10 \%$ of sputtered tungsten forms dust in a steady state reactor, it constitutes a serious safety issue. Low $\mathrm{Z}$ liquid plasma facing materials offer an alternative with much less impact due to plasma contamination.

\section{Simulation codes and parameters}

The code UEDGE [6] is used in ARIES RS geometry [16]. The simulation region is shown in fig.1. The density at the inner flux surface boundary (at $1.25 \mathrm{~cm}$ inside the separatrix at the outer midplane) is set at $5 \times 10^{19} / \mathrm{m}^{3}$. The heating power into the SOL is taken to be $100 \mathrm{MW}$.

Conventional values of the density diffusion coefficient and thermal diffusivity are used: $.33 \mathrm{~m}^{2} / \mathrm{s}$ and $.5 \mathrm{~m}^{2} / \mathrm{s}$, respectively. A high wall recycling coefficient of $99 \%$ is used, as is appropriate for a steady state device. Higher recycling coefficients produced results which are very close, but convergence is sometimes much slower.

A radiating impurity, Neon, is included in the simulations, to reduce the peak divertor heat load. For simplicity, the impurity concentration is taken to be a constant fraction of the electron density. Unless otherwise noted, the impurity fraction was adjusted to produce a reactor relevant peak heat flux on the divertor plate of $8 \mathrm{MW} / \mathrm{m}^{2}$ 


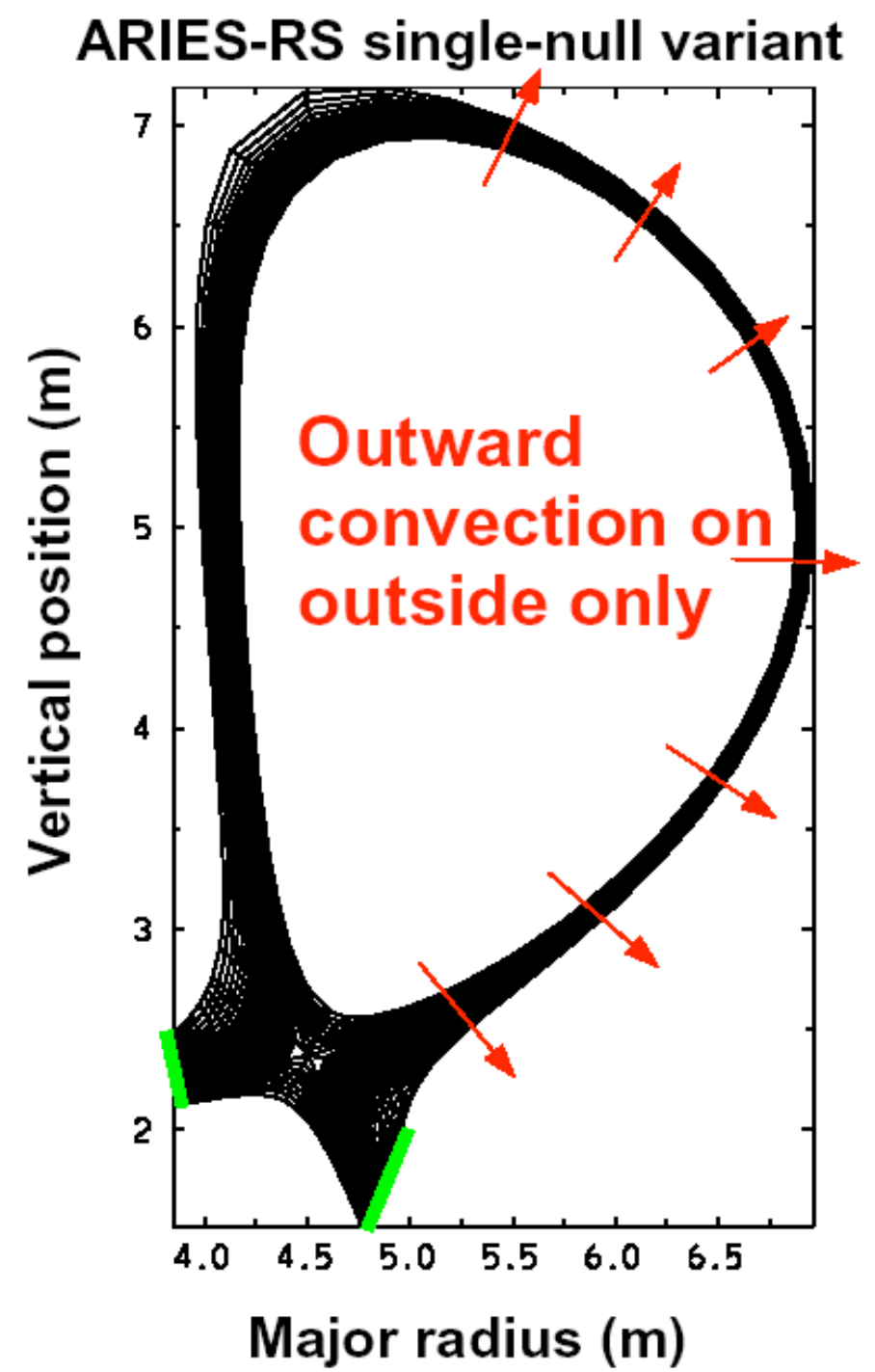

Fig.1. The computational domain of the UEDGE calculations

The neutral transport code NU' ' [13] solves the full integral equations to obtain the steady state neutral distribution function both in space and velocity, and can solve 3-D geometries. Charge exchange and ionization are included, along with finite density corrections. NUT has been benchmarked against experimental tokamak data on C-mod and TEXT $[14,15]$. Plasma profiles of the density, electron temperature and ion temperature are input, along with the recycling source at the wall. These are provided as output by UEDGE.

In this paper, we only compute the neutral distribution at the outer midplane. To simplify the neutral calculations we simplify the computational geometry in NUT in a way appropriate for the outer mid-plane. Over $90 \%$ of the neutrals impacting the wall arise from the region within $10 \mathrm{~cm}$ of the edge of the plasma. For ARIES RS, the radii of 
curvature of the plasma on the outer midplane in the poloidal and toroidal direction are much longer than this $(200 \mathrm{~cm}$ and $560 \mathrm{~cm})$. Thus, the flux could be computed with a slab approximation. For slightly better accuracy, we take the computational geometry to be cylindrical, with a curvature chosen to match the poloidal curvature of ARIES RS on the midplane. Since the poloidal variation of the plasma quantities is also small in the region which determines the neutral flux at the outer midplane, for simplicity we take the plasma quantitites to be independent of poloidal position as well for the neutral calculation.

Finally, NUT has only been implemented with one plasma species, so we approximate the $50 \% \mathrm{D}-50 \% \mathrm{~T}$ plasma as a single species with atomic mass 2.5 . To compute the sputtering, we use the Bohdansky formula, and average the fitting parameters for $\mathrm{D}$ and $\mathrm{T}$ to obtain a hybrid sputtering coefficient for mass 2.5 particles. The resulting hybrid sputtering coefficient, over the energy range which produces the dominant sputtering for cases of interest here, is shown in fig. 2. Effects of oblique incidence are approximately included by multiplying the normal incidence formula by a factor of 2 , as in reference [5].

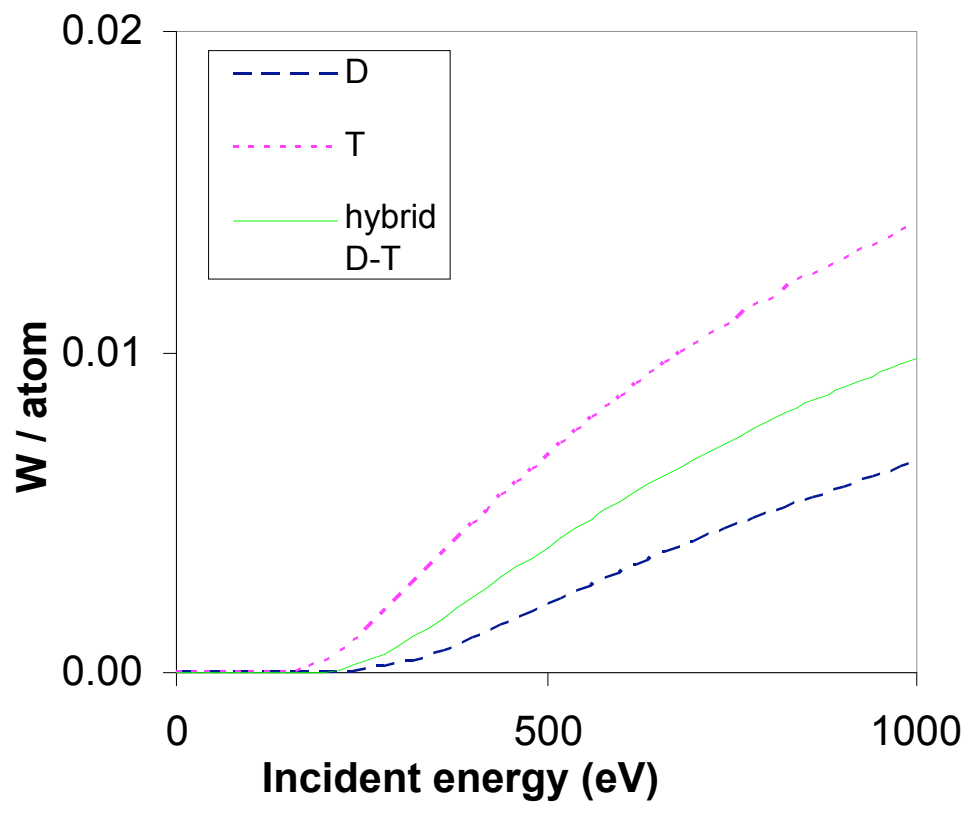

Fig.2. Sputtering coefficients of tungsten for D, T and the hybrid case

NUT requires that plasma profiles be specified which are beyond the range of those computed by UEDGE. For this purpose, we presume H-mode like profiles, and values characteristic of a reactor, with a pedestal temperature, density and width inside the SOL of $4 \mathrm{keV}, 1.7 \times 10^{20}$, and $5 \mathrm{~cm}$, respectively. The density profile in the plasma is assumed to be nearly flat (a conventional assumption for H-modes), with an edge value equal to $85 \%$ of the Greenwald limit, and a volume averaged density peaking of 1.2 . The central temperature is taken to be $24 \mathrm{keV}$, as is discussed in more detail in section III. 


\section{Convection model}

Convection has been included in UEDGE to successfully model DIII-D edge plasmas for reactor relevant operating modes: ELMy $\mathrm{H}$-modes with a highly radiative divertor [7]. The best fit to the data was obtained by choosing a convection profile which increases from $10 \mathrm{~m} / \mathrm{s}$ at the separatrix to $137 \mathrm{~m} / \mathrm{s}$ in the far SOL (at the midplane) with a roughly exponential profile. Data from C-mod and DIII-D also indicate a roughly exponential increase of convection with distance [17]. Thus, we will use an exponential convection profile here.

The use of such a profile on DIII-D enabled the far SOL plasma density profiles to be matched, as well as the $\mathrm{D}_{\square}$ recycling light and neutral pressure [7]. When the convection term was left out, UEDGE under-predicted the main chamber recycling and wall fluxes by about a factor of 10-15 (although reflected light from other locations might account for some of this discrepancy). Experimental results from ASDEX are consistent with convection values in the far SOL of $40-80 \mathrm{~m} / \mathrm{s}$ [3], as are C-mod [18] and DIII-D [19] results. It is reasonable to conjecture that similar values could be present in a reactor. There is also reason to be concerned that the neglect of such convection in the far SOL could lead to an underestimation of the plasma-wall interaction in a reactor projection.

To better estimate the possible convection magnitude in a reactor, we consider three characteristics of experimental SOL data: 1) the density scale length in the region around $\mathrm{r} / \mathrm{a}=1.02-1.042$ ) the ratio of the SOL density at $\mathrm{r} / \mathrm{a}=1.04$ to the value at the separatrix and 3) the plasma flux impinging on the wall. We choose convection magnitudes for our reactor simulations to roughly match these three characteristics of present experiments.

On many experiments, the SOL density profile decay rate in the far SOL is substantially slower than the decay rate near the separatrix. More precisely, the density scale length in the far SOL is much longer than the decay scale length near the sepratrix. This has been noted on many experiments, such as ASDEX, ASDEX UG [3], Alcator C-mod [18], DIII-D [19], JT-60 and JT-60U [20]. This is one indicator that plasma transport is increasing with distance from the separatrix. The change in decay length generally occurs at a distance $\mathrm{d}$ from the separatrix (normalized to the minor radius a) of $\mathrm{d} / \mathrm{a} \sim .02$. Without including convection, this feature is not reproduced by UEDGE for our reactor simulations.

In fig. 3 the decay scale length for experimental data between $\mathrm{d} / \mathrm{a}=.02$ and $\mathrm{d} / \mathrm{a}=.04$ is shown verses the normalized density. The definition of normalized density here is the line average density divided by the Greenwald density $\mathrm{n}_{\mathrm{G}}$. Though there is considerable scatter in the experimental data, the decay scale length is longer for increasing normalized density. Tokamak reactors operate with a high normalized density ( $\sim 1$ for ARIES RS). 


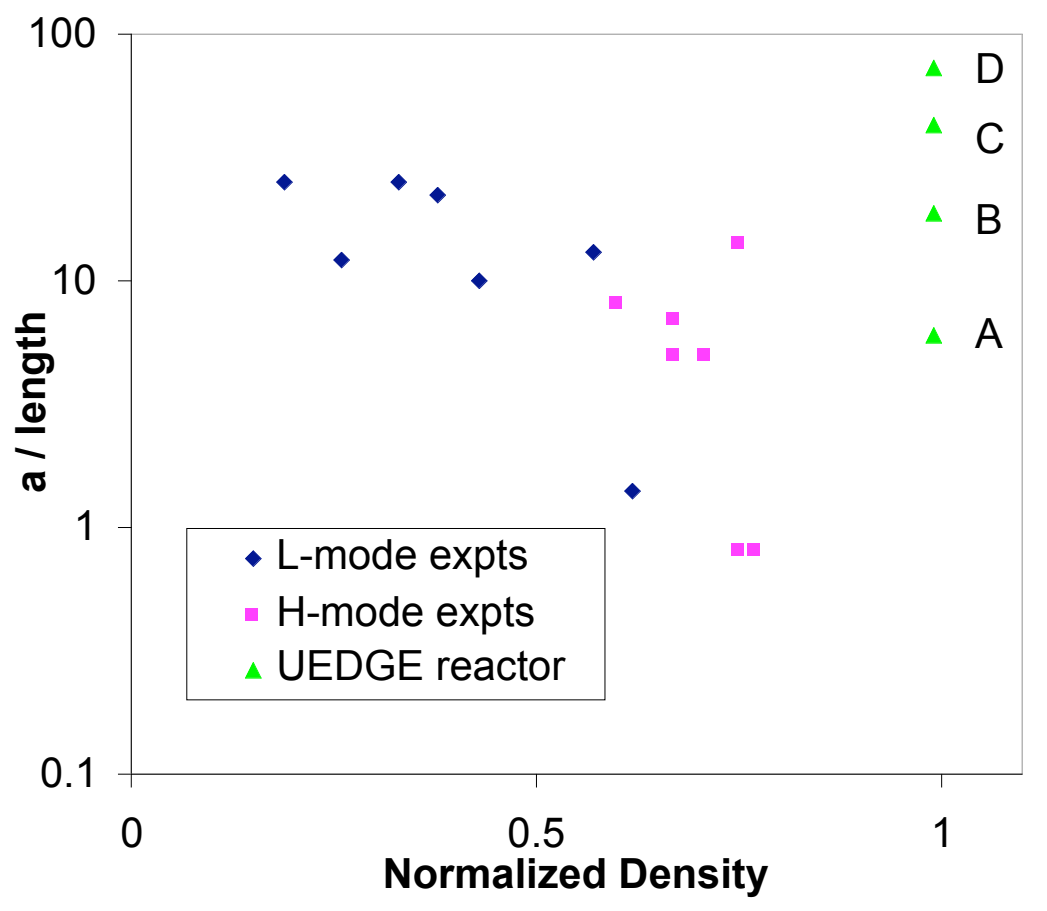

Fig.3. The inverse density scale length of the SOL for $\mathrm{d} / \mathrm{a}=.02$ .04 from experiments and from UEDGE verses the plasma density normalized to $\mathrm{n}_{\mathrm{G}}$

Also shown are results from UEDGE simulations for four cases. As noted above, the convection increases exponentially from the value at $\mathrm{d} / \mathrm{a}=-.01$ to reach the value at $\mathrm{d} / \mathrm{a}=.045$, so these two values alone determine the profile.

\begin{tabular}{|l|l|l|}
\hline Case & Convection at $\mathrm{d} / \mathrm{a}=-.01$ & Convection $\mathrm{at} \mathrm{d} / \mathrm{a}=.045$ \\
\hline A & 10. & 100. \\
\hline B & 10. & 50. \\
\hline C & 5. & 100. \\
\hline D & 0. & 0. \\
\hline
\end{tabular}

For the standard model without convection (case D), the UEDGE results are well outside the range of experimental data. Case A seems closest to the expectation for a high normalized density, case $\mathrm{B}$ is within the experimental range but does not follow the trend with normalized density. Case C is marginally outside the data and clearly does not follow the trend, and case $\mathrm{D}$ is well outside the data. Thus, decreasing convection below the values in case $\mathrm{A}$ at either $\mathrm{d}=-.01$ or $\mathrm{d}=.045$ reduces the apparent degree of agreement with existing data.

Another parameter which is obviously important for plasma-wall interaction is the plasma density at the wall. In our reactor simulations, this is at $\mathrm{d} / \mathrm{a}=.045$. We plot the ratio the plasma density at $\mathrm{d} / \mathrm{a}=.04$ to the separatrix density verses normalized density. 
As can be seen, the experimental data forms a clear trend toward higher SOL density ratios at higher normalized density. Again, case A appears to match the data trends best, case $\mathrm{D}$ is well outside the data, and case $\mathrm{B}$ and $\mathrm{C}$ are intermediate. Again, decreasing convection below the values in case $\mathrm{A}$ at either $\mathrm{d}=-.01$ or $\mathrm{d}=.045$ reduces the apparent degree of agreement with existing data.

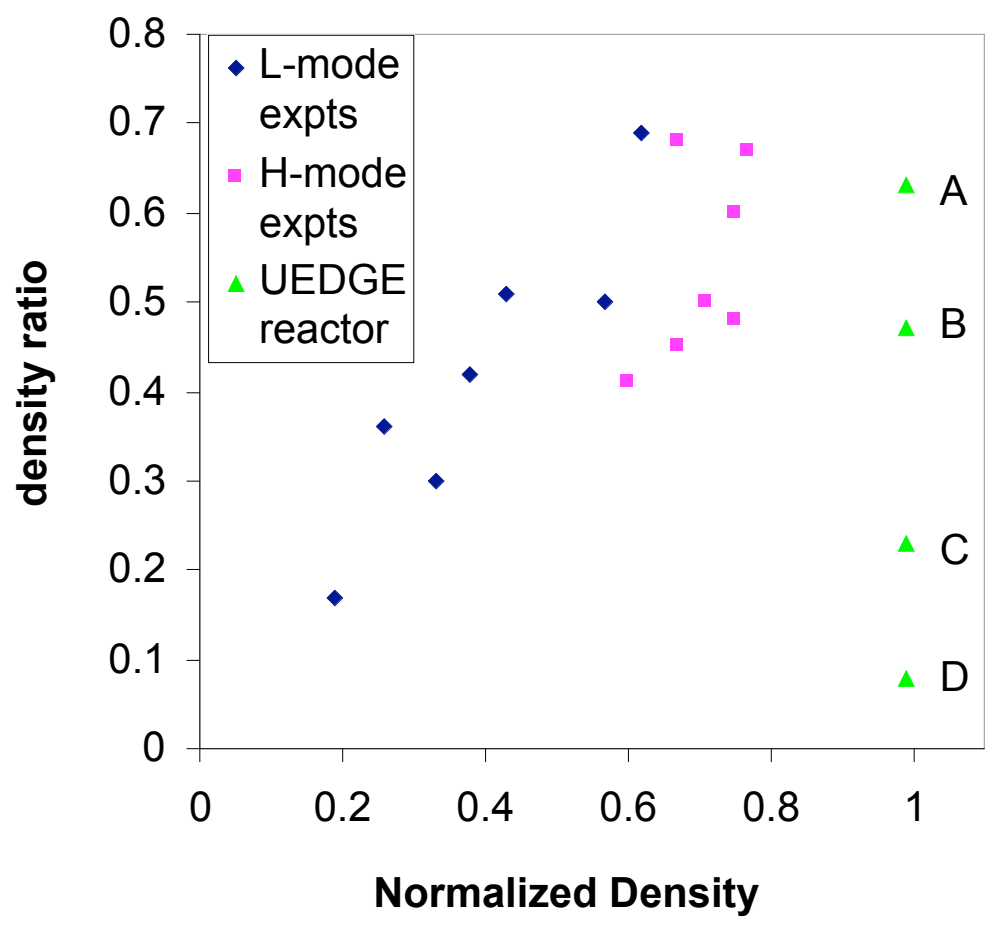

Fig 4 . The ratio of the SOL density at $\mathrm{d} / \mathrm{a}=.04$ to the separatrix density from experiments and UEDGE verses plasma density normalized to $\mathrm{n}_{\mathrm{G}}$

The plasma flux at the wall position is obviously another important indicator of plasmawall interaction. The DIII-D analysis found that, without convection, the plasma wall flux was lower by a factor of 10-15 compared to experimental data, and convection brought the flux into agreement for a convection $\sim 100 \mathrm{~m} / \mathrm{s}$ near the wall. For our reactor case, we now consider which convection magnitude best matches experimental trends.

Experimental data for the flux $\square$ has been compiled by Labombard et. al. for over a hundred shots from Asdex Upgrade, C-mod, JET and JT-60. Labombard et. al. note that the data shows a trend where wall flux increases roughly quadratically with density, according to the relation

$\square_{\mathrm{L}}=10^{21} / \mathrm{m}^{2} \mathrm{~s}\left(\mathrm{n}_{\mathrm{e}} / 10^{20} / \mathrm{m}^{3}\right)^{2}$

where $\mathrm{n}_{\mathrm{e}}$ is the line averaged electron density. However, data from different diagnostics was included in this graph, and some points are up to an order of magnitude from this 
line. For a reactor with $\mathrm{N} \sim 2 \times 10^{20} / \mathrm{m}^{3}$ the wall flux according to the trend above is $\square_{\mathrm{L}}=$ $4 \times 10^{21} / \mathrm{m}^{2}$ s. In fig. 5 below, we plot the fluxes divided by $\square_{\mathrm{L}}$ for the data above as well as the UEDGE cases. There is considerable scatter in the data, but case A and B are within the data, case $\mathrm{C}$ is clearly somewhat low and the case $\mathrm{D}$ without convection is outside the data.

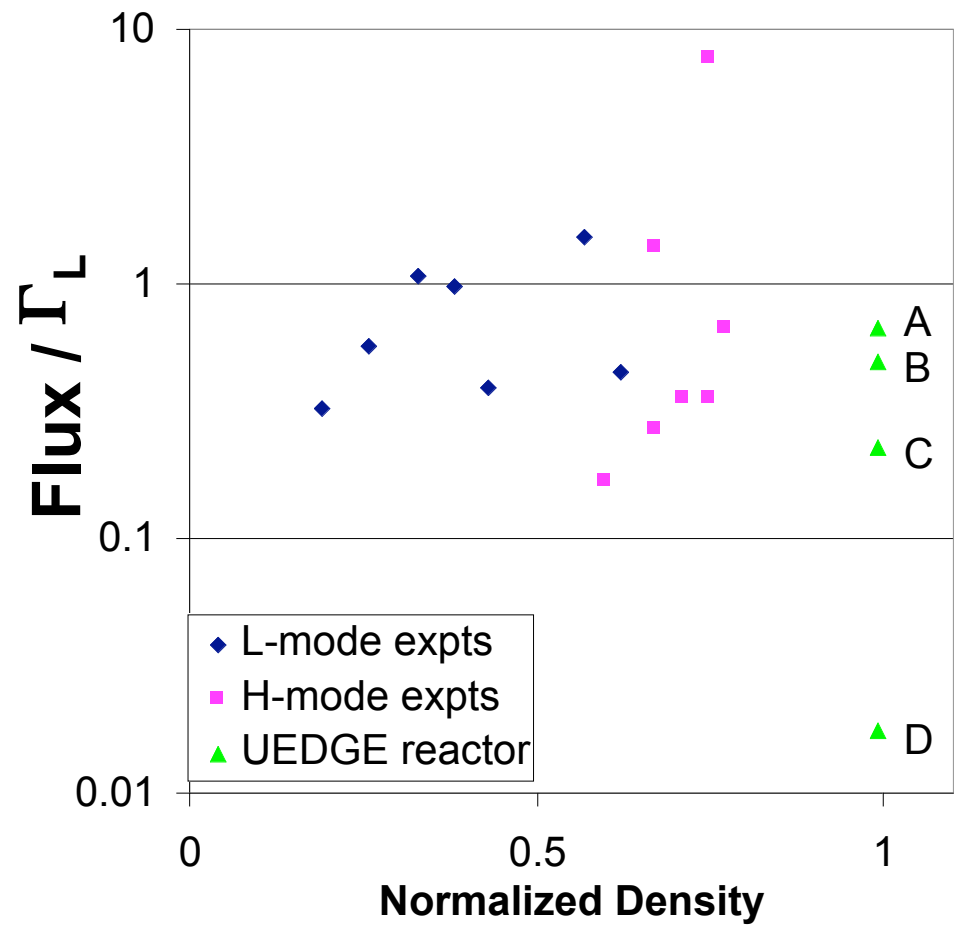

Fig.5. The plasma wall flux normanlized to $\square_{\mathrm{L}}$ for experiments and UEDGE verses density normalized to $\mathrm{n}_{\mathrm{G}}$

Thus, we conclude that case A, with a convection profile of $100 \mathrm{~m} / \mathrm{s}$ at the wall is most consistent with the data for all three SOL characteristics. The case with no convection is outside the range of all the experimental characteristics examined above. Case B, with 50 $\mathrm{m} / \mathrm{s}$ convection at the wall is next most consistent, and case $C$ is marginally within the range of the data but does not follow the apparent trends with normalized density.

We now consider the reactor implications of cases A through D.

\section{Code results}

Plasma-wall interactions have been examined by Behrisch et. al. [21] for an ITER-like case using B2-EIRENE and a conventional transport model without convection. They report a similar outboard flux, $7 \times 10^{19} / \mathrm{m}^{2} \mathrm{~s}$, as in our ARIES-RS like case without convection, $4 \times 10^{19} / \mathrm{m}^{2} \mathrm{~s}$. As might be expected, our cases with convection will produce a substantially larger erosion rate than is estimated in that work. 
The plasma parameters in the cases with and without convection are given below. The output from UEDGE was used in NUT to compute the C-X neutral spectrum striking the wall.

\begin{tabular}{|ll|l|l|l|l|}
\hline $\begin{array}{l}\text { Parameter } \\
\text { (outer midplane wall) }\end{array}$ & Case A & Case B & Case C & Case D \\
\hline Plasma wall flux & $\left(10^{20} \mathrm{~m}^{-2} \mathrm{~s}^{-1}\right)$ & 32. & 11. & 10. & .36 \\
\hline Density & $\left(10^{20} \mathrm{~m}^{-3}\right)$ & .3 & .2 & .08 & .02 \\
\hline Ion temperature $\quad(\mathrm{eV})$ & 20 & 29 & 53 & 103 \\
\hline Electron temperature $(\mathrm{eV})$ & 12 & 16 & 18 & 20 \\
\hline Ne fraction & .0022 & .0022 & .005 & .0095 \\
\hline C-X neutral flux $\quad\left(10^{20} \mathrm{~m}^{-2} \mathrm{~s}^{-1}\right)$ & 6.8 & 2.3 & 2.4 & 0.10 \\
\hline Average C-X neutral energy $(\mathrm{eV})$ & 186 & 234 & 422 & 619 \\
\hline
\end{tabular}

The plasma flux generates a source of recycled neutrals at the wall. These neutrals penetrate into the plasma, and generate new hot neutrals by charge exchange with the plasma. This charge exchange flux is a significant fraction of the wall source $(\sim 20-30 \%)$, and includes high energy neutrals from charge exchange deep in the plasma. The case with convection has a much higher plasma flux, which leads to a larger $\mathrm{C}-\mathrm{X}$ flux back to the wall. However, the higher wall density in the convection case also tends to shield the penetrating neutrals from the higher temperature regions of the plasma, leading to a significant reduction in the average neutral energy. This in turn reduces the sputtering coefficient of high $\mathrm{Z}$ materials.

We note that calculations by Behrisch et. al. for an ITER-like geometry were performed with a conventional SOL transport model. They found the plasma midplane flux to the wall was $7 \times 10^{19}$, the C-X neutral flux was $\sim 3 \times 10^{19}$, and the average CX energy was $\sim 600 \mathrm{eV}$. These results are similar to case $\mathrm{D}$ above.

We now turn to the sputtering consequences for various wall materials.

\section{Sputtering results for solid walls: tungsten}

As in previous calculations, sputtering for high $\mathrm{Z}$ materials is predominantly due to high energy charge-exchange neutrals. Sputtering from direct plasma interaction effects is significant only for the case without convection. To compute the sputtering from direct plasma interaction, we use an energy distribution corresponding to a Maxwellian with the ion temperature, with three times the electron temperature added.

\begin{tabular}{|l|l|l|l|l|}
\hline $\begin{array}{l}\text { W Sputtering neglecting re- } \\
\text { deposition (mm/yr) }\end{array}$ & Case A & Case B & Case C & Case D \\
\hline Direct plasma interaction & .0002 & .003 & .07 & 0.021 \\
\hline W sputtering C-X neutrals & 0.6 & 0.27 & 0.49 & 0.049 \\
\hline Total W sputtering & 0.6 & 0.27 & 0.56 & 0.07 \\
\hline
\end{tabular}


We presume the tungsten density is $95 \%$ of the theoretical maximum. As can be seen, the convection in cases $\mathrm{A}, \mathrm{B}$ and $\mathrm{C}$ increases the erosion by roughly an order of magnitude over case D without convection. Behrisch et. al found a total sputtering rate of $.22 \mathrm{~mm} / \mathrm{yr}$, which is about three times the result here without convection, and a third of the case here with convection.

An additional example of the weak dependence of the tungsten sputtering is shown in fig. below, where the neon concentration was varied. This peak heat flux on the divertor plate also varied strongly, but the tungsten sputtering rate varied relatively weakly.

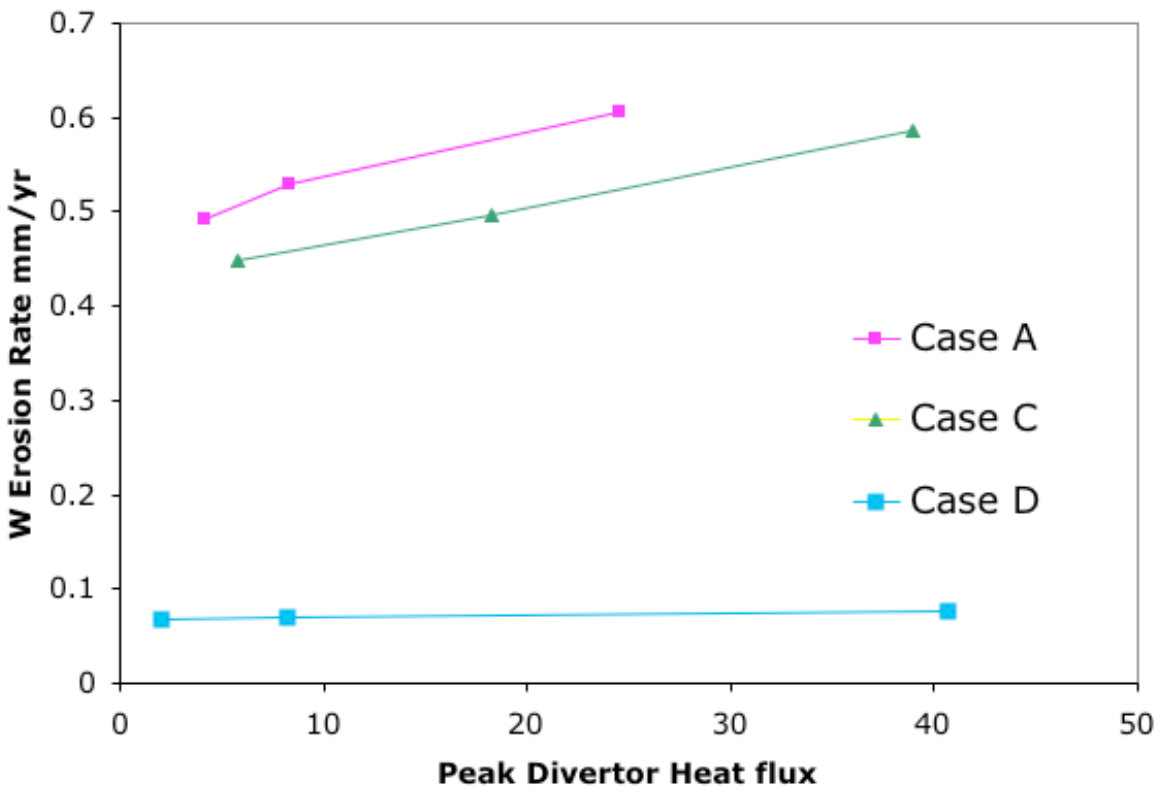

Fig. 6. The erosion rate for a tungsten wall verses the peak divertor heat flux, by varying the neon concentration

The prompt re-deposition rate for tungsten is expected to be modest at best. Prompt redeposition occurs when a sputtered $\mathrm{W}$ atom is ionized in the plasma, and the ion trajectory brings it back to the wall within a gyro-orbit. For the case with convection, the density at the wall of the SOL is still low enough that the gyro-radius is about half of the mean free path for ionization, and is much longer than the sheath width. This is different from divertor regions, where the densities are much higher, and prompt re-deposition of high $\mathrm{Z}$ atoms is strong. Impurity re-deposition from plasma transport is difficult to estimate when transport is dominated by blob-like convection, as will be discussed below.

\section{Potential implications of tungsten sputtering on plasma operation}


High Z plasma impurities can seriously affect plasma energy confinement at low concentrations, due to excessive radiative energy loss. We define $\square$ as the impurity shielding factor, i.e., the ratio of impurities entering the plasma to the impurities emitted from the wall. For the present parameters, virtually all of the impurities will be ionized in the SOL before they reach the separatrix. Thus, plasma transport processes will be crucial in determining the actual plasma contamination. The inward impurity flux in the presence of strong blob-like transport is not well understood, as will be indicated in section 7 . Therefore, we consider the screening factors which are found in present experiments, where most of the impurities are also ionized in the SOL layer.

The tokamak Alcator $\mathrm{C}$-mod has walls of high $\mathrm{Z}$ material (molybdenum), and has found $\square$ to be in the range of .1-.02 for H-mode discharges [22] for molybdenum generated from the antenna shields. These shields are at a distance $\sim 1.5 \mathrm{~cm}$ from the plasma, which is at a similar $\mathrm{d} / \mathrm{a}$ to our first wall. On $\mathrm{C}-\mathrm{mod}$, the molybdenum mean free path is only $\sim 2 \mathrm{~mm}$, so virtually all of the Mo is ionized in the SOL, as in our reactor case. ASDEX has found penetration values $\sim 1 \%$ for inboard tungsten walls [23]. The $\mathrm{W}$ concentration in the plasma for ASDEX would be acceptable for a reactor. However, edge plasma temperatures in ASDEX are several times lower than in reactors, so the large majority of C-X neutrals on ASDEX are well below the sputtering threshold of W. This is not the case for a reactor, so the low W concentration on ASDEX cannot be directly extrapolated. In addition to experiments, UEDGE has been used to examine plasma contamination by Sn, another high Z impurity, but neglecting convection [24]. This leads to $\square$ values roughly estimated to be in the range of $\sim .1$.

For H-mode-like plasma profiles, we plot the effect on the required Lawson confinement parameter of different $\square$ values in fig 7. Radiation from bremsstrahlung, $\mathrm{Ne}, \mathrm{He}$ and $\mathrm{W}$ is included, as well as fuel dilution. We assume a $10 \% \mathrm{He}$ fraction (similar to ARIES RS). We have taken the high $\mathrm{Z}$ plasma impurity confinement time $\square$ to equal the energy confinement time from plasma energy losses (a conventional assumption similar to ARIES RS), and plasma tungsten density $n_{W}$ is computed by equating the source rate and the loss rate

$\mathrm{n}_{\mathrm{W}} \mathrm{V} / \mathrm{G}=\mathrm{S}_{\mathrm{W}} \mathrm{A} \square$

where $S_{W}$ is the tungsten wall source in $\mathrm{m}^{-2} \mathrm{~s}^{-1}, \mathrm{~V}$ is the plasma volume, and $\mathrm{A}$ is the area of the tungsten source. Here, we use ARIES RS values, with $V=349 \mathrm{~m}^{3}$ and $A=218 \mathrm{~m}^{2}$, which is half the wall surface area (we presume strong convection only occurs on the outboard side). External energy inputs in addition to alpha power are included (e.g. current drive), with an assumed plasma Q of 27, as in ARIES RS.

The allowable tungsten concentration is sensitive to plasma temperature and density profiles. We choose a profiles characteristic of H-modes, with fairly flat density $(\mathrm{n}(0) /<\mathrm{n}>=1.2)$ a pedestal temperature of $4 \mathrm{kev}$ (a midrange estimate for ITER) and a ratio of the core temperature to the pedestal temperature $\mathrm{T}(0) / \mathrm{T}(\mathrm{ped})=5.5$. The latter value is characteristic of experimental values for H-modes in the ITER profile database [25] for shots with a ratio of the ion temperature to electron temperature $<1$. (This 
condition is conventionally presumed to pertain in a reactor due to preferential heating of the electrons by alpha particles.) Results are shown in fig. 7.

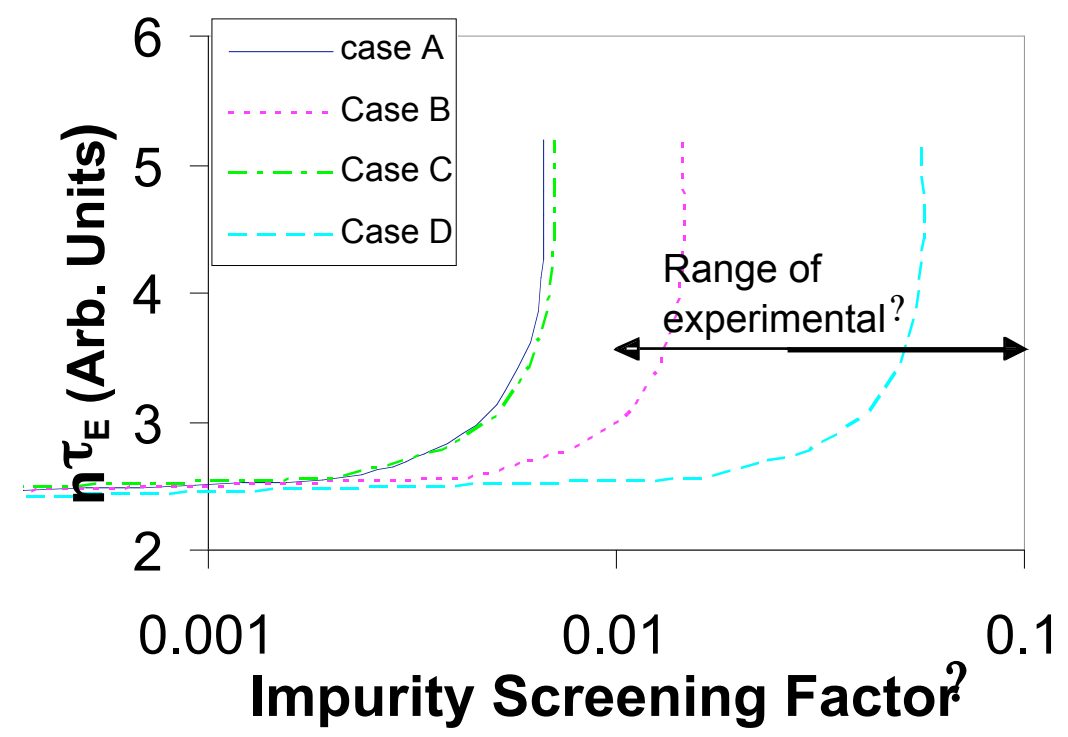

Fig.7. The required Lawson parameter $\mathrm{n} \square$ for the confinement time from plasma losses verses the impurity screening factor

For a given impurity generation rate, there is a critical $\square$ value beyond which the impurity radiation makes plasma sustainment impossible for any level of plasma confinement. This critical $\square$ value decreases sharply with decreasing core temperature, so if this value is exceeded at an initial temperature, the plasma will evolve to a radiation collapse. As can be seen, $\square$ values found in present experiments and simulations, $.01<\square<.1$, would make plasma operation impossible with the $\mathrm{W}$ flux of the convection case $\mathrm{A}$ and $\mathrm{C}$, and is borderline for case B. Even below this critical value, the plasma energy confinement requirement can be increased substantially, which is a serious feasibility issue. For case D (with no plasma convection), there is substantial overlap between the acceptable range of $\square$ and the present experimental values.

Impurity transport in the SOL is not well understood in the presence of blob-like transport, so projections of $\square$ to a reactor are quite uncertain. Nonetheless, there is clearly reason to be seriously concerned about the impact on plasma operation of enhanced $\mathrm{W}$ influx due to SOL convection.

\section{Potential safety consequences of tungsten dust}

In addition to structural consequences, erosion can lead to dust formation. This is a poorly understood process, but analysis for ITER very roughly estimate that $10 \%$ of sputtered material might form dust. With the erosion rate of case A for the outboard first wall, this amounts to $240 \mathrm{~kg} / \mathrm{yr}$ of dust generated. Assuming a wall lifetime of two years, $480 \mathrm{~kg}$ of dust could accumulate. In the event of a loss of vacuum accident accident, this 
dust could result in releases by atmospheric suspension. Also, the dust increases the W surface area available for oxidation and thus mobilization. The amount of dust accumulated after 2 years would have a surface greater than the first wall area by roughly 2-3 orders of magnitude.

Analysis of W releases for the EVOLVE design [26] found that the dosage at site boundary was $0.13 \mathrm{~Sv} / \mathrm{kg}$ for stacked releases of $\mathrm{W}$ under worst case atmospheric conditions, and about $2 \%$ of the tungsten might be released to the environment without filtering. After two years of dust accumulation, this could lead to an offsite dosage of $1.25 \mathrm{~Sv}$, which is well above the no evacuation limit of .01 Sv. With an assumed filtering efficiency of $99 \%$, this drops to marginally above the no evacuation limit (.012 Sv).

Given the uncertainties in the dust generation calculation above, tungsten dust generation might pose a significant safety issue.

\section{Sputtering consequences for liquid walls}

Structural erosion and dust generation are not relevant issues for liquid plasma facing components. However, impurity contamination of the plasma is an issue. The acceptable core concentration of low $\mathrm{Z}$ impurities is about three orders of magnitude greater than for high $\mathrm{Z}$ impurities. But low $\mathrm{Z}$ solid plasma facing components are not considered for reactors because the structural erosion rates and dust generation rates are unacceptable. The use of liquid materials which can be continuously replenished offers the possibility of using low $\mathrm{Z}$ plasma facing components. Examples would be flibe, Li and SnLi alloys. Note that the sputtering from $\mathrm{SnLi}$ is very similar to sputtering from pure $\mathrm{Li}$ [28], since a layer of pure Li forms at the surface which is several atoms thick due to thermodynamic considerations. Experiments and simulations show that $\mathrm{Sn}$ is effectively not sputtered. Also, SnLi does not have the hydrogen gettering, vapor pressure or chemical reactivity of pure Li. It is a low recycling material with sputtering properties close to pure Li. We do not consider pure liquid $\mathrm{Li}$ here, since the gettering characteristis of pure $\mathrm{Li}$ would probably strongly modify the SOL, so that our empirical extrapolations are invalid.

Sputtering data for flibe [29], SnLi [28] and Sn [30] at 45 degree incidence were used. In the case of $\mathrm{SnLi}$, we have presumed that the fraction of sputtered Li which is emitted as an ion $(60 \%)$ is immediately re-deposited, and thus can be neglected.

\begin{tabular}{|l|l|l|l|}
\hline $\begin{array}{l}\text { Material sputtering } \\
\text { rate }\left(10^{19} / \mathrm{m}^{2} \mathrm{sec}\right)\end{array}$ & Case A & Case D & $\begin{array}{l}\text { acceptable } \\
\text { for case A }\end{array}$ \\
\hline Flibe: & & & .05 \\
\hline $\mathrm{Li}$ & 4. & .02 & \\
\hline $\mathrm{Be}$ & 2. & .03 & \\
\hline $\mathrm{F}$ & 3. & .08 & \\
\hline SnLi: $\mathrm{Li}$ & 16 & .2 & .05 \\
\hline $\mathrm{Sn}$ & 0.4 & .04 & .008 \\
\hline
\end{tabular}


For low $\mathrm{Z}$ impurities such as $\mathrm{Li}, \mathrm{Be}$ and $\mathrm{F}$, concentration limits would be set by fuel dilution. We assume that a reduction of the D-T density by $10 \%$ is acceptable. Since tin is a high $\mathrm{Z}$ material, the tolerable core fraction of $\mathrm{Sn}$ is determined by radiation. An acceptable fraction of $\mathrm{Sn}$ has been estimated to be $0.02 \%$ [27]. Note that the corresponding acceptable penetration factor for $\mathrm{Sn}$ is about the same as for $\mathrm{W}$.

The acceptable penetration factors for the low $\mathrm{Z}$ materials are about an order of magnitude higher than for the high $\mathrm{Z}$ materials $\mathrm{W}$ and $\mathrm{Sn}$, and are in the range of experimental penetration factors. Thus, liquid low $\mathrm{Z}$ materials may be necessary for satisfactory plasma operation.

These estimates do not account self-consistently for the reduction of the electron temperature near the wall owing to ionization and excitation of the sputtered impurities. It is shown in Ref. 31 using conventional diffusive transport only that a kind of plasma power-detachment can occur at the main-chamber wall as the input impurity flux is increase. Such a mechanism would reduce the wall sheath potential and thus ion sputtering, but not the neutral sputtering. The low electron temperatures of a few eV may also affect the character of the blobs near the wall.

\section{Consequences of blob-like dynamics on impurity transport}

Sputtered impurities which are not promptly re-deposited within a gyro-orbit can be redeposited by plasma transport processes near the wall, thereby preventing plasma contamination. Blob-like transport renders this process more complex than can be described with either a simple diffusion model or a convection model [32]. The spaces between blobs have relatively lower plasma density, and are convected inwards toward the core. Thus, impurities ionized in these regions are transported to the main plasma much more quickly than in a conventional model, after which they should undergo transport similar to the main-plasma hydrogen. At present, there is no way to predict the fraction of impurities which will be ionized in these low density regions, the velocity at which they will be convected inward, or their mean free path before they disintegrate into the background turbulence. Some transport modeling comparing inward and outward convection of impurities in present-day experiments has been recently performed [33]. Further research is required for this important and complex subject, before appropriate models of impurity transport in blob-dominated regimes can be formulated.

\section{Conclusions}

The empirically motivated convection model for developed here for SOL transport clearly has uncertainties when projected to a reactor. Nonetheless, we have shown that the neglect of convection for reactor cases leads to SOL profiles and plasma fluxes to the wall which appear inconsistent with present experimental data. This is particularly true for operation at a high fraction of the Greenwald density, as is anticipated for reactors. The model adopted here brings those parameters into the range expected from present experiments. 
This model results in a substantial increase in the plasma-wall interaction for reactor cases. Plasma fluxes to the wall increase by nearly two orders of magnitude due to the inclusion of convection. However, convection (together with a very high recycling coefficient) also lowers the temperature of the far SOL, which somewhat mitigates the resulting increase in sputtering.

The sputtering rates of high $\mathrm{Z}$ walls ( $\mathrm{W}$ and $\mathrm{Sn}$ ) are increased by about an order of magnitude, and are dominated by the high energy $\mathrm{C}-\mathrm{X}$ neutrals. Sputtering from low- $Z$ liquids is increased by about one and a half orders of magnitude. Both high $\mathrm{Z}$ and low $\mathrm{Z}$ impurities will be ionized in the SOL, and the contamination of the bulk plasma will be dominated by plasma transport effects in the SOL.

There is a considerable uncertainty in extrapolation impurity screening in the presence of strong blob-like transport from present experiments to reactors. However, for screening factors in the range of present experiments, impurities from high $\mathrm{Z}$ walls such as $\mathrm{W}$ and Sn would probably preclude ignited plasma operation. Low Z liquid materials (flibe or $\mathrm{SnLi}$ ) are compatible with reactor plasma operation for screening factors in the present experimental range.

In light of these results, two lines of research need to be pursued to resolve this potentially show stopping issue: 1) the physics of SOL transport, including both the outward convection of the bulk species and the inward convection of impurities, and 2) the development of low $\mathrm{Z}$ liquid plasma facing technologies, to provide a viable alternative to high $\mathrm{Z}$ plasma facing materials.

Furthermore, the plasma convection could also increase the amount of dust generated by solid $\mathrm{W}$ walls to the point where it becomes a significant safety issue, also meriting further investigation.

Acknowledgements: The author would like to thank B. Merril and P. Thomas for helpful discussions. This work was performed under the auspices of the U.S. Department of Energy by contracts DE-FG03-96ER54346 at the University of Texas and W-7405-Eng48 at University of California Lawrence Livermore National Laboratory.

[1] B. LaBombard, M.V. Umansky, R.L. Boivin et.al., Nucl. Fusion 40 (2000) 2041

[2] J.A. Boedo, D.L. Rudakov, R.J. Colchin. Et. al., J. Nucl. Mater. 313-316 (2003) 813

[3] J. Neuhauser, D. Coster, et.al., Plasma Phys. Control. Fusion 44 (2002) 855

[4] R. Behrisch, G. Federici, A. Kukushkin, et. al., J. Nucl. Mater. 313-316 (2003) 388

[5] G. Federici, J.N. Brooks, D.P. Coster, et. al., J. Nucl. Mater. 290-293 (2001) 260

[6] T.D. Rognlien, J.L. Milovich, M.E. Rensick et.al., J. Nucl. Mater. 196-198 (1992) 34

[7] A. Yu. Pigarov, S.I. Krasheninnikov, T.D. Rognlien, et. al., Phys. Plasmas 9 (2002) 1287

[8] S.J. Zweben, D.P. Stotler, J.L. Terry et. al., Phys. Plasmas 9 (2002) 1981

[9] M.V.A.P. Heller, Z.A. Brasilio, et.al., . Plasmas 6 (1999) 846 
[10] S.I. Krasheninnikov Phys. Lett. A 283 (2001) 368

[11] D. A. D'Ippolito, J. R. Myra, S. I. Krasheninnikov, Phys. Plasmas 9 (2002) 222

[12] S.I. Krasheninnikov, S. A. Galkin, A. Yu. Pigarov, et. al., $29^{\text {th }}$ EPS Conference on Controlled Fusion and Plasma Physics, Montreux, 26B III (2002) 205

[13] P. M. Valanju, J. Comp. Phys. 88, 114 (1990).

[14] R. D. Bengtson, P. M. Valanju, A. Ouroua, and W. L. Rowan, Rev. Sci. Instrum. 61, 3110 (1990), J. A. Boedo, R. D. Bengtson, A. Ouroua, and P. M. Valanju, Rev. Sci.

Instrum. 59, 1494 (1988), and W. L. Rowan, C. C. Klepper, C. P. Ritz, R. D. Bengtson, K. W. Gentle, P. E. Phillips, T. L. Rhodes, B. Richards, and A. J. Wootton, Nucl. Fusion 27, 1105 (1987).

[15] B. C. Stratton, R. J. Fonck, A. T. Ramsey, E. J. Synakowski, B. Grek, K. W. Hill, D. W. Johnson, D. K. Mansfield, H. Park, G. Taylor, and P. M. Valanju, Nucl. Fusion 30, 675 (1990).

[16] F. Najmabadi et. al., Fusion Eng. \& Des. 38 (1997) 3-25

[17] http://itpa.ipp.mpg.de/coordinating_committee/meeting_2002 03/

Soldiv itpaCCv4(10 25).pdf

[18] B. LaBombard, R.L. Boivin, et. al., Phys. Plasmas 8 (2001) 2107

[19] http://web.gat.com/exp/2003/exchange/outgoing/yer/boedo_2002psi.pdf

[20] N. Asakura, N. Hosogane, et. al., J. Nucl. Mater. 266-269 (1999) 182

[21] R. Behrisch, G. Federici, et. al., J. Nucl. Mater. 313-316 (2003) 389

[22] B. Lipshultz, D.A. Pappas, B. LaBombard et. al., J. Nucl. Mater. 290-293 (2001) 286

[23] R. Neu, R. Dux, A. Geier, et. al., Plasma Phys. and Control. Fusion, 44 (2002) 811

[24] T.D. Rognlien, M.E. Rensink, Contrib. Plasma Phys. 42 (2002) 2-4, 193

[25] M. Kotschenreuther et. al., Proceedings of the $16^{\text {th }}$ IAEA Fusion Energy Conference, Montreal, Canada IAEA-CN-64/D1-5, 1996

[26] B. J. Merrill, et al.,"Safety Evaluation of the EVOLVE Blanket Concept," 15th Topical Meeting on the Technology of Fusion Energy, Washington, DC, November 17-21, 2002, to be published in Fusion Science and Technology.

[27] J. Wesson, Tokamaks, $2^{\text {nd }}$ edition, (Clarendon, Oxford 1997) p 103

[28] J.P. Allain, D. N. Ruzic, M.R. Hendricks, J. Nucl. Mater. 290-293 (2001) 33

[29] J.P. Allain, private communication

[30] M.D. Coventry, J.P. Allain, D. N. Ruzic, J. Nucl. Mater. 313-316 (2003) 636

[31] T.D. Rognlien, M.E. Rensink, Phys. Plasmas 9 (2002) 2120.

[32] S.I. Krasheninnikov, S.A. Galkin, A. Yu. Pigarov et al., $29^{\text {th }}$ EPS Conf. Controlled Fusion Plasma Physics, Montreux, Switzerland, P-3.205 (2002).

[33] A.Yu. Pigarov, S.I. Krasheninnikov, T.D. Rognlien et al., $30^{\text {th }}$ EPS Conf. Controlled Fusion Plasma Physics, St. Petersburg, Russia, P-3.170 (2003). 